\title{
The New Approach to Solving the Riemann Boundary Value Problem with Infinite Index
}

\section{R. B. Salimov, E. N. Karabasheva}

Kazan State University of Architecture and Engineering, 1, Zelenaya str., 420043, Kazan, Tatarstan, Russia, salimov@5354.ru, enkarabasheva@bk.ru

This research considers Riemann - Hilbert boundary value problem with infinite index where edge condition of problem is established by the real axis. To solve this problem the approach based on the removal of the infinite discontinuity of the argument of boundary condition coefficient is used. The approach is analogous to the one which, in the context of the finite index of the problem in researches by F. D. Gakhov, helps to remove a discontinuity of initial genre of boundary condition coefficient with specially created functions, different from the ones in this research.

Key words: Riemann boundary value problem, analytical function, infinite index.

\section{References}

1. Muskhelishvili N. I. Singular integral equations. Moscow, Nauka, 1968, 511 p. (in Russian).

2. Gakhov F. D. Boundary value problems. Moscow, Nauka, 1977, 640 p. (in Russian).

3. Govorov N. V. Riemann's boundary problem with infinite index. Moscow, Nauka, 1986, 239 p. (in Russian). 4. Tolochko M. E. About the solvability of the homogeneous Riemann boundary value problem for the half-plane with infinite index. Izvestiya Akad. Nauk BSSR. Ser. Fiz.-mat. nauki, 1972, no. 5, pp. 34-41 (in Russian).

5. Sandrygailo I. E. On Hilbert - Rieman boundary value problem for half-plane with infinite index. Izvestiya Akad. Nauk BSSR. Ser. Fiz.-Mat. Nauki, 1974, no. 6, pp. 872875 (in Russian).

6. Monahov V. N., Semenko E. V. Boundary value problem with infinite index in Hardy spaces. Dokl. Akad. Nauk SSSR, 1986, vol. 291, no. 3, pp. 544-547 (in Russian).

7. Alehno A. G. Sufficient conditions for the solvability of homogeneous Riemann boundary value problem

with infinite index. Trudy matematicheskogo tsentra imeni N. I. Lobacheuskogo, Kazan, 2002, vol. 14, pp. 7177 (in Russian).

8. Garifianov F. N. About a special case of the Riemann problem. Trydu seminara po kraevum zadacham. Kazan, 1984, no 22, pp. 66-68 (in Russian).

9. Katc B. A. About Riemann problem with an oscillating coefficient. Trydu seminara po kraevum zadacham. Kazan, 1977, no. 14, pp. 110-120 (in Russian).

10. Salimov R. B., Shabalin P. L. The regularizing factor method for solving a homogeneous Hilbert problem with an infinite index. Russian Math. [Izvestiya VUZ. Matematika], 2001, vol. 45, iss. 4, pp. 74-77.

11. Markushevich A. I. The theory of analytic functions : in 2 vol. Moscow, Nauka, 1968, vol. 2, 624 p. (in Russian).

12. Levin B. Ya. Distribution of zeros of entire functions. Moscow, Gostechizdat, 1956, 632 p. (in Russian).

13. Salimov R. B., Shabalin P. L. Solution of the Hilbert Problem with infinite index. Math. Notes, 2003, vol. 73, iss. 5, pp. 680-689. DOI: 10.4213/mzm221.

УДК 517.538 .7

\section{РАСХОДИМОСТЬ ВСЮДУ ПРОЦЕССОВ ЛАГРАНЖА НА ЕДИНИЧНОЙ ОКРУЖНОСТИ}

\author{
С. В. Тышкевич ${ }^{1}$, А. В. Шаталина ${ }^{2}$ \\ ${ }^{1}$ Кандидат фризико-математических наук, доцент кафредры теории фуннкций и приближений, Саратовский государственный \\ университет им. Н. Г. Чернышевского, tyszkiewicz@yandex.ru \\ ${ }^{2}$ Кандидат фризико-математических наук, доцент кафредры теории фрункций и приближений, Саратовский государственный \\ университет им. Н. Г. Чернышевского, teh-plast@bk.ru \\ Изучаются вопросы сходимости интерполяционных процессов Лагранжа в замкнутом единичном круге. Выбор матрицы с \\ определённым распределением узлов интерполирования позволил построить множество, полностью покрывающее еди- \\ ничную окружность, и срункцию, для которой процесс расходится всюду на этом множестве.
}

Ключевые слова: интерполирование, полиномы Лагранжа.

Пусть $A C$ - множество функций $f(z)$, аналитических в $|z|<1$ и непрерывных в $|z| \leqslant 1$, с обычным модулем непрерывности $\omega(f, \delta)$ и равномерной нормой; $\Omega-$ множество функций $\omega$ типа модуля непрерывности, $\Omega_{0}-$ функции $\omega \in \Omega$, для которых

$$
\lim _{\delta \rightarrow 0+} \frac{\delta}{\omega(\delta)}=0 .
$$


Введем множества:

$$
A C(\omega)=\{f \in A C ; \omega(f, \delta)=O(\omega(\delta))\}, \quad A C^{*}(\omega)=\{f \in A C ; \omega(f, \delta)=o(\omega(\delta))\}
$$

где $\omega \in \Omega-$ наперед заданная функция. Для матрицы узлов интерполирования $M=\left\{z_{k, n}\right\}_{k=0, n=1}^{n-1, \infty}$, заданных на $|z|=1$, построим интерполяционный процесс Лагранжа:

$$
L_{n}(M, f, z)=\sum_{k=0}^{n-1} f\left(z_{k, n}\right) l_{k, n}(M, z)
$$

где $l_{k, n}(M, z)=\frac{\omega_{k, n}(z)}{\left(z-z_{k, n}\right) \omega_{k, n}^{\prime}(z-k, n)}, \omega_{k, n}(z)=\prod_{k=0}^{n-1}\left(z-z_{k, n}\right)$.

Хорошо известно [1], что в случае матрицы равноотстоящих узлов для любой $f(z) \in A C$ имеет место равномерная сходимость $L_{n}(M, f, z)$ к $f(z)$ внутри $|z|<1$. Спрашивается, как ведут себя интерполяционные процессы на $|z|=1$ ? В работе [2] были найдены необходимые и достаточные условия, которым должна удовлетворять $\omega(\delta)$, для того чтобы имела место равномерная сходимость $L_{n}(M, f, z)$ для $f(z) \in A C(\omega)$ на $|z|=1$, была найдена метрическая характеристика множеств точек расходимости этих процессов на $|z|=1$. Позднее в [3] строились функции $f(z) \in A C(\omega)$ и $f(z) \in A C^{*}(\omega)$, для которых процессы Лагранжа расходятся почти всюду. В данной работе уточняется последний результат. А именно доказывается следующая теорема.

Теорема. Пусть $M$ - матриц̧а, каждая n-я строка которой является корнями $n$-й степени из -1. Если $\omega \in \Omega_{0}$ удовлетворяет условию

$$
\varlimsup_{n \rightarrow \infty} \omega\left(\frac{1}{n}\right) \ln n>0,
$$

тогда существует функция $f \in A C(\omega)$, что интерполяционный процесс Лагранжа расходится всюду на $|z|=1$, причем

$$
\varliminf_{n \rightarrow \infty}\left\|L_{n}(M, f, \cdot)-f\right\|_{A C}=0 .
$$

Если же для $\omega \in \Omega_{0}$ справедливо

$$
\varlimsup_{n \rightarrow \infty} \omega\left(\frac{1}{n}\right) \ln n=\infty,
$$

тогда существует $f \in A C^{*}(\omega)$, для которой интерполяционный процесс Лагранжа неограниченно расходится всюду на $|z|=1$, причём справедливо (1).

Перед доказательством теоремы приведем несколько лемм.

Лемма 1. Пусть $\left\{n_{p}\right\}_{p=1}^{\infty}-$ последовательность простьх иисел. Тогда для любого $\varepsilon, 0<\varepsilon<1$, можно указать число $q=q(\varepsilon)>2$, что для каждого натурального числа н существует нату-

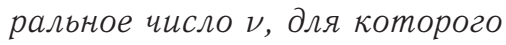

$$
\varepsilon \cdot \sum_{p=\mu}^{\nu} \frac{1}{n_{p}}>2 \pi, \quad n_{\nu}<n_{\mu}^{q}
$$

Доказательство. Необходимо воспользоваться следующим асимптотическим равенством для последовательности простых чисел:

$$
\sum_{n_{p} \leq x} \frac{1}{n_{p}}=\ln \ln x+a+O\left(\frac{1}{\ln x}\right),
$$

где $a-$ абсолютная константа [4].

Замечание 1. Пусть $M$ - матрица, $n$-я строка которой является корнями $n$-й степени из -1 ; $\left\{n_{p}\right\}, \mu, \nu, q, \varepsilon$ удовлетворяют условиям леммы 1. Тогда для всех $i, j, \mu \leq i \neq j \leq \nu$, и для всех индексов $k, 0 \leq k \leq n_{i}-1$ и $s, 0 \leq s \leq n_{j}-1$, за исключением двух $k^{*}$ и $s^{*}$, для которых узлы $z_{k^{*}, n_{i}}=z_{s^{*}, n_{j}}=\{-1\}$, справедливо

$$
\left|z_{k, n_{i}}-z_{s, n_{j}}\right|>n_{\mu}^{-2 q}
$$


Предположим, что это не так, т. е. существуют $l, t: 0 \leq\left(k^{*}+l\right) \leq n_{i}-1,0 \leq\left(s^{*}+t\right) \leq n_{j}-1$, такие, что $\left|z_{k^{*}+l, n_{i}}-z_{s^{*}+t, n_{j}}\right| \leq n_{\mu}^{-2 q}$. Так как $z_{k, n_{p}}=\exp \left(i \pi \frac{2 k-1}{n_{p}}\right)$ последнее равенство перепишется:

$$
\begin{aligned}
& n_{\mu}^{-2 q} \geq 2\left|\sin \left(\frac{\pi}{2}\left(\frac{2\left(k^{*}+l\right)-1}{n_{i}}-\frac{2\left(s^{*}+t\right)-1}{n_{j}}\right)\right)\right| \geq \\
& \geq \frac{2}{\pi}\left|\frac{2\left(k^{*}+l\right)-1}{n_{i}} \pi-\frac{2\left(s^{*}+t\right)-1}{n_{j}} \pi\right|=\frac{2}{\pi}\left|\frac{2 \pi l}{n_{i}}-\frac{2 \pi t}{n_{j}}\right| .
\end{aligned}
$$

Отсюда:

Получили противоречие.

$$
\frac{1}{4 \pi n_{\mu}^{2 q}} \geq \frac{1}{\pi}\left|\frac{l}{n_{i}}-\frac{t}{n_{j}}\right| \geq \frac{1}{\pi n_{\mu}^{2 q}}
$$

Лемма 2. Пусть $M \subset\{|z|=1\}$ - матрица равноотстоящих узлов, $r>2$ - действительное число, $l \in \mathbb{N}$ - фиксировано. Тогда для каждого $n \in \mathbb{N}, n>l$, можно построить множество

$$
F_{n, r}=\bigcup_{k=1}^{n}\left[\exp \left(i\left(\theta_{k-1, n}+a\right)\right), \exp \left(i\left(\theta_{k, n}-a\right)\right)\right], \quad a=\frac{\theta_{k, n}-\theta_{k-1, n}}{r},
$$

линейная мера которого теs $F_{n, r}=2 \pi-\frac{4 \pi}{r}$ такое, что для всех $z \in F_{n, r}$ выполняется неравенство

$$
\sum_{k=0}^{n-1}\left|l_{k, n}(M, z)\right| \geq \frac{1}{\pi r} \ln \left(\frac{n}{l}\right)
$$

где итрих означает отсутствие произвольных l слагаемых.

Доказательство приведено в работе [3].

Лемма 3. Пусть $M \subset\{|z|=1\}, l,\left\{n_{p}\right\}, \mu, \nu, q-$ такие, как описано выше. Тогда для каждого $n_{\mu} \in\left\{n_{p}\right\}$ найдутся $n_{\nu_{0}}, \nu_{0}<\nu$, множество $E_{\mu} \subset\{|z|=1\}$ и конечное семейство дуг $\Delta_{t_{j}, \mu+j}^{0}$, $0 \leq j \leq \nu_{o}-\mu$, чmo

a) $\operatorname{mes} E_{\mu} \geq 2 \pi-2$;

б) $E_{\mu} \subset \bigcup_{j=0}^{\nu_{0}-\mu} \Delta_{t_{j}, \mu+j}^{0}$;

в) для любой точки $z \in E_{\mu} \cap \Delta_{t_{j}, \mu+j}^{0}$ справедливо $L_{n_{\mu+j}}(M, l, z) \geq c_{1} \ln \left(n_{\mu+j} / l\right)$, где $c_{1}=$ const.

Доказательство леммы 3 подробно изложено в работе [3], приведём здесь лишь его схему.

Множество $E_{\mu}$ строилось за $\nu_{0}-\mu$ шагов. На каждом $(j-1)$-м шаге, $1 \leq j \leq \nu_{o}-\mu$, было построено множество, состоящее из $j$ непересекающихся дуг $E_{\mu} \cap \Delta_{t_{p}, n_{\mu+p}}^{0} \cap F_{n_{\mu+p, r}}, 0 \leq p \leq j,\left(F_{n_{\mu+p, r}}\right.$ из леммы 2), которые лежат на дуге единичной окружности от точки $\exp \left(i\left(\theta_{t_{0}, n_{\mu}}+\frac{\theta_{t_{0}+1, n_{\mu}}-\theta_{t_{0}, n_{\mu}}}{r}\right)\right)$ до точки $\exp \left(i\left(\theta_{t_{j-1}+1, n_{\mu+j-1}}-\frac{\theta_{t_{j-1}+1, n_{\mu+j-1}}-\theta_{t_{j-1}, n_{\mu+j-1}}}{r}\right)\right)$, где $t_{0}$ выбрано произвольно из условия $0 \leq t_{0} \leq n_{\mu}$, а $r>2-$ фиксировано.

На следующем $j$-м шаге к построенному множеству присоединяли либо:

а) целую дугу

$$
\left[\exp \left(i\left(\theta_{t_{j}, n_{\mu+j}}+a\right)\right), \exp \left(i\left(\theta_{t_{j}+1, n_{\mu+j}}-a\right)\right)\right], \quad a=\frac{\theta_{t_{j}+1, n_{\mu+j}}-\theta_{t_{j}, n_{\mu+j}}}{r},
$$

если мера наименьшей дуги $\gamma_{j}=\left(z_{t_{j-1}+1, n_{\mu+j-1}} ; z_{t_{j}, n_{\mu+j}}\right)$ не превосходила $\frac{1}{n_{\mu+j}}$; причем дуга $\gamma_{j}$ не входила в множество $E_{\mu}$, т. е. при построении множества $E_{\mu}$ на $j$-м шаге выбросили дугу единичной окружности, мера которой не больше $\alpha_{j}=\left\{\frac{1}{n_{\mu+j}}+\frac{2 \pi}{r n_{\mu+j}}+\frac{2 \pi}{r n_{\mu+j-1}}\right\}$, а узел $z_{t_{j-1}+1, n_{\mu+j-1}}$ принадлежит выброшенной дуге;

б) частичную дугу

$$
\left[\exp \left(i\left(\theta_{t_{j-1}+1, n_{\mu+j-1}}+\frac{\theta_{t_{j-1}+2, n_{\mu+j-1}}-\theta_{t_{j-1}+1, n_{\mu+j-1}}}{r}\right)\right), \exp \left(i\left(\theta_{t_{j}+1, n_{\mu+j}}-a\right)\right)\right],
$$


если мера $\gamma_{j}$ больше $\frac{1}{n_{\mu+j}}$. В этом случае выбрасывали дугу единичной окружности меры, не большей $\left[\frac{2 \pi}{r n_{\mu+j}}+\frac{2 \pi}{r n_{\mu+j-1}}\right]<\alpha_{j}$, и опять тот же узел принадлежит выброшенной дуге. После построения множества $E_{\mu}$ проверяется выполнение пунктов а), б) из условия, а с учетом леммы 2 получаем выполнение пункта в).

Лемма 4. Для любого $\mu \in \mathbb{N}$ существует $\nu \in \mathbb{N}$, что для любой точки $z \in\{|z|=1\}$ найдется номер $t_{j} \in\left\{n_{\mu}, 2 n_{\mu}, \ldots, n_{\nu}, 2 n_{\nu}\right\}$, при котором

$$
\sum_{k=0}^{n_{j}-1}\left|l_{k, n_{j}}(M, z)\right| \geq C \ln t_{j}
$$

где C-абсолютная константа, а итрих у знака $\sum$ означает отсутствие одного произвольного слагаемого.

Доказательство. Положим $r=24 \pi$. Заметим, что строки с номерами $n_{\mu}, n_{\mu+1}, \ldots, n_{\nu}$ не имеют ни одного общего узла, за исключением точки -1 . Более того, в силу замечания 1 расстояние между любыми другими двумя узлами любых двух строк из этой пачки не меньше, чем $n_{\mu}^{-2 q}$. Строки с номерами $n_{k}$ и $2 n_{p}, \mu \leq k, p \leq \nu$, вообще не имеют общих узлов. По лемме 3 для номеров $n_{\mu}$ и $n_{\nu}$ существуют $\nu_{0}<\nu$, множество $E_{\mu}$, конечное семейство дуг $\Delta_{t_{j}, \mu+j}^{0}$, для которых выполняются пункты а), б), в); причем можно положить $l=1$. Для каждого «выброшенного» из множества $E_{\mu}$ узла $z_{t_{j}+1, n_{\mu+j}}, j=\overline{0, \nu_{0}-\mu}$ (см. доказательство леммы 3 ) найдем дугу $\Delta_{t_{j}^{*}, 2 n_{\mu+j}}^{0}$, такую, что этот узел будет серединой найденной дуги. Получим еще одно семейство дуг:

$$
\delta_{t_{j}^{*}, 2 n_{\mu+j}}=\left[\exp \left(i\left(\theta_{t_{j}^{*}, 2 n_{\mu+j}}+a\right)\right), \exp \left(i\left(\theta_{t_{j}^{*}+1,2 n_{\mu+j}}-a\right)\right)\right], \quad a=\frac{1}{r}\left(\theta_{t_{j}^{*}+1,2 n_{\mu+j}}-\theta_{t_{j}^{*}, 2 n_{\mu+j}}\right) .
$$

Обозначим это семейство $A$. Так как

$$
\frac{1}{2} \operatorname{mes} \delta_{t_{j}^{*}, 2 n_{\mu+j}}=\frac{\pi}{2 n_{\mu+j}}-\frac{\pi}{r n_{\mu+j u}}=\beta_{j},
$$

$r=24 \pi$, то очевидно, что $\beta_{j}>\alpha_{j}$, где $\alpha_{j}$ из леммы 3 . То есть дуги, входящие в семейство $A$ и во множество $E_{\mu}$, полностью покрывают единичную окружность, и для любой точки $z \in\{|z|=1\}$ найдется индекс $t_{j}(z), t_{j} \in\left\{n_{\mu}, 2 n_{\mu}, \ldots, n_{\nu}, 2 n_{n u}\right\}$, такой, что в силу лемм 2 и 3 имеем (3).

Пусть везде в дальнейшем $\left\{n_{p}\right\}, \mu, \nu, q-$ такие, как мы определили выше. Числа $W_{k, n_{i}}$, $0 \leq\left|W_{k, n_{i}}\right| \leq 1$ - произвольные, причем если для каких-то узлов из матрицы $M$ не выполняется условие (2), то считаем соответствующее число $W_{k, n_{i}}=W_{s, n_{j}}=0$.

Лемма 5. Пусть даны $\left\{n_{p}\right\}, \mu, \nu, q, W_{k, n_{i}}$. Тогда существует функиия $f \in A C(\omega,|z| \leq 1)$ такая, что

$$
f\left(z_{k, n_{t}}\right)=W_{k, n_{t}}+O\left(\frac{1}{n_{\mu}^{2 q}}\right)
$$

причем

$$
\begin{aligned}
& \max _{z \in|z| \leq 1}|f(z)| \leq 2, \\
& \omega(f, \delta) \leq 9 n_{m u}^{q^{79 q}} \delta .
\end{aligned}
$$

Доказательство. Приведем схему доказательства.

Введем функцию

$$
P_{k, n_{t}}^{s_{k}}(z)=\left[\exp \left(i\left(\pi-\theta_{k, n_{t}}\right)\right) z+2\right]^{s_{k}},
$$

где $0 \leq k \leq n_{t}, \mu \leq t \leq \nu$.

Выбираем показатель степени $s_{k}$ так, чтобы

$$
\left|\Psi_{k, n_{t}}\left(\exp ^{i \varphi}\right)\right|=\left|\frac{l_{k, n_{t}}(\exp (i \varphi))}{P_{k, n_{t}}^{s_{k}}(\exp (i \varphi))}\right| \leq \begin{cases}1, & \varphi \in \Delta_{k, t} \\ \frac{1}{n_{\mu} 5 q}, & \varphi \notin \Delta_{k, t}\end{cases}
$$

где $\Delta_{k, t}=\left[\varphi_{k, n_{t}}-\frac{1}{n_{t}^{16 q}}, \varphi_{k, n_{t}}+\frac{1}{n_{t}^{16 q}}\right]$. 
Для этого достаточно положить $s_{k}=n_{t}^{38 q}$. Это легко проверяется, если использовать свойства функции $P_{k, n_{t}}(z)$ и арифметические преобразования. В ходе проверки, пользуясь интегральной формулой Коши, оценками полиномов на линиях уровня, принципом максимума модуля аналитической функции получаем:

$$
\left|\Psi_{k, n_{t}}(z+h)-\Psi_{k, n_{t}}(z)\right|<9|h| n_{t}^{78 q} .
$$

Положим теперь:

$$
f(z)=\sum_{t=\mu}^{\nu} \sum_{k=o}^{n_{t}-1}{ }^{\prime} W_{k, n_{t}} \cdot \Psi(z)
$$

где штрих у знака суммы означает, что отсутствуют слагаемые, для которых $W_{k, n_{t}}=0$.

Очевидно, что функция $f(z)$ - аналитическая в $|z|<1$ и непрерывная в $|z| \leq 1$. Подсчитав значение $f(z)$ в произвольном фиксированном узле $z_{k_{0}, n_{j}}$, будем иметь:

$$
f\left(z_{k_{0}, n_{j}}\right)=W_{k_{0}, n_{j}}+\sum_{k=0}^{\nu} \sum_{k=0}^{n_{t}-1}{ }^{\prime \prime} W_{k, n_{t}} \cdot \Psi_{k, n_{t}}\left(z_{k_{0}, n_{j}}\right)
$$

второй штрих у знака суммы означает отсутствие слагаемого с индексом $\left(k_{0}, n_{j}\right)$. Теперь, учитывая оценки (7), (8), выбор точек $W_{k, n_{t}}$ и того факта, что мы имеем не более чем $n_{\mu}^{q}$ строк, а значит, не более чем $n_{\mu}^{2 q}$ узлов, получаем (4)-(6).

Лемма 6. Пусть $M-$ матрица равноотстоящих узлов, $n_{p_{0}}-$ произвольная строка, $\Delta_{j, p}^{0}-$ одна из частинных дуг, $z_{0}=\exp \left(i \varphi_{0}\right) \in \Delta_{j, p}^{0}$. Положим

$$
W_{k, n_{p}}=\left[\eta_{k, n_{p}} \cdot \exp \left(-\frac{i k \pi}{n_{p}}\right)\right]
$$

где числа

$$
\eta_{k, n_{p}}=\operatorname{sign} \sin \left(\frac{1}{2}\left(\varphi_{0}-\theta_{k, n_{p}}\right)\right) .
$$

Тогда для всех $z \in \Delta_{j, p}^{0}$ справедливо:

$$
\left|\sum_{k=0}^{n_{p}-1} W_{k, n_{p}} \cdot l_{k, n_{p}}(M, z)\right|=L_{n_{p}}(M, z)=\sum_{k=0}^{n_{p-1}}\left|l_{k, n_{p}}(M, z)\right| .
$$

Доказательство следует из представления

$$
l_{k, n_{p}}\left(M, z_{0}\right)=\frac{1}{n} \cdot \exp \left(\frac{i}{2}\left(n_{p}-1\right)\left(\varphi_{0}-\theta_{0, n_{p}}\right)\right) \cdot \sin \left(\frac{n}{2}\left(\varphi_{0}-\theta_{0, n_{p}}\right)\right) \cdot \frac{\exp \left(\frac{i k \pi}{n_{p}}\right)}{\sin \left(\frac{1}{2}\left(\varphi_{0}-\theta_{k, n_{p}}\right)\right)}
$$

и равенств (8).

Замечание 2. Рассмотрим множество $E_{\mu}$ из леммы 3. Как уже говорилось, оно состоит из конечного $\left(\nu_{0}-\mu\right)$ числа непересекающихся открытых дуг. На каждой такой дуге зафиксируем по точке $z_{p}=\exp \left(i \varphi_{p}\right), 0 \leq p \leq \nu_{0}-\mu-1$, определим числа $W_{k, n_{p}}$ по формулам (9). Тогда в силу леммы 5 существует функция $f_{\mu}(z)$ со свойствами (4)-(6). Кроме того, для любого $z \in E_{\mu}$ существует $j(z)$, $0 \leq j \leq \nu_{0}-\mu$, такой, что выполняется в) из леммы 3. Отсюда и условия (4):

$$
\left|L_{n_{\mu+p}}\left(M, f_{\mu}, z\right)\right|=\left|\sum_{k=0}^{n_{\mu+p}-1} W_{k, n_{p+\mu}} \cdot l_{k, n_{p+\mu}}(M, z)\right|+\sum_{k=0}^{n_{p-1}} O\left(n_{\mu}^{-2 q}\right) \geq C_{1} \ln \left(n_{\mu+p}\right)-C_{2}\left(n_{\mu}^{-q}\right),
$$

$C_{1}, C_{2}-$ абсолютные константы.

Лемма 7. Пусть $M-$ матрица корней $n$-й степени из -1 . Тогда каждого $\mu, n_{\mu} \in \mathbb{N}$ существует функция $f_{\mu} \in A C$ такая, ито справедливо (5),(6), и для любой точки $z \in\{|z|=1\}$ найдется номер $n_{t} \in\left\{n_{\mu}, 2 n_{\mu}, \ldots, n_{\nu}, 2 n_{\nu}\right\}$, для которого справедливо:

$$
\left|L_{n_{t}}\left(M, f_{\mu}, z\right)\right| \geq C^{*} \cdot \ln n_{t}-C^{* *} n_{\mu}^{-q},
$$

где $C^{*}, C^{* *}-$ абсолютные константы. 
Доказательство. В ходе доказательства леммы 4 строились две системы дуг: дуги, входящие во множество $E_{\mu}$, и дуги, перекрывающие $\{|z|=1\} \backslash E_{\mu}$, т. е. $A$. На дугах из $E_{\mu}$ зафиксируем произвольную точку и определим числа $W_{k, n_{t}}$ как показано в замечании 2 (если какой-то узел равен -1 , тогда полагаем соответствующую точку $\left.W_{k, n_{t}}=0\right)$. На дугах семейства $A$ выбираем и фиксируем центральную точку (см. доказательство леммы 4) и по ней, аналогично (9), определяем числа $W_{k, 2 n_{j}}$. Заметим, что расстояние между узлами из пачки $\left\{n_{\mu}, 2 n_{\mu}, \ldots, 2 n_{\nu}\right\}$ не меньше $\frac{1}{2 n_{\mu}^{2 q}}$ (см. замечание 1). Дословно повторяя рассуждения леммы 5 , получаем, что существует функция $f_{\mu}(z)$ со свойствами (4)-(6). Осталось проверить (11). Если $z \in\{|z|=1\} \backslash\{-1\}$, то $z$ принадлежит либо $E_{\mu}$, либо $A$. В силу рассуждений, приведенных в замечании 2 , оценка (11) становится очевидной.

В работе [3] была доказана следующая лемма.

Лемма 8. Пусть $M=\{|z|=1\}-$ матрица равноотстоящих узлов и $\omega \in \Omega_{0}$.

Если для любого фиксированного $j$ существуют последовательности: $\left\{\beta_{k, j}\right\}_{k=1}^{\infty}-$ положительных чисел; $\left\{\mu_{k, j}\right\}$ и $\left\{\nu_{k, j}\right\}-$ натуральных чисел; $\left\{f_{k, j}\right\}-$ функций, аналитических в $|z|<1$ и непрерьвньх в $|z| \leq 1 ;\left\{E_{k, j}\right\}-$ измеримых множеств, которые удовлетворяют

a) $\lim \beta_{k, j}=0, \lim \mu_{k, j}=\infty, \mu_{k, j} \leq \nu_{k, j}$;

б) $\left\|f_{k, j}\right\|_{A C} \leq \omega\left(\beta_{k, j}\right)$;

в) $\omega\left(f_{k, j}, \delta\right) \leq \frac{\omega\left(\beta_{k, j}\right)}{\beta_{k, j}} \delta$;

г) $E_{k, j} \in\{|z|=1\}$, mes $E_{k, j} \geq 2 \pi$, для любого $z \in E_{k, j}$ существует $l, \mu_{k, j} \leq l \leq \nu_{k, j}$, ито

$$
\left|L_{l}\left(M, f_{k, j}, z\right)\right| \geq C_{3}(j)>0,
$$

где $C_{3}=$ const.

Тогда существуют функция $f \in A C(\omega)$ и множество $E$, mes $E=2 \pi$, такие, ито

$$
\varlimsup_{n \rightarrow \infty}\left|L_{n}(M, f, z)-f(z)\right|>0
$$

везде на Е, причем

$$
\varliminf_{n \rightarrow \infty}\left|L_{n}(M, f, z)-f(z)\right|=0 .
$$

Доказательство теоремы. Пусть выполняются условия теоремы 1 . Для любых $j \in \mathbb{N}$, при каждом натуральном $k$ положим: $\mu_{k, j}=n_{\mu}, \nu_{k, j}=n_{\nu} ; \beta_{k, j}=\beta_{\mu}=\left[9\left(n_{\mu}^{q}\right)^{79 q}\right]^{-1} ; f_{k, j}=f_{\mu}=\frac{\omega\left(\beta_{k, j}\right)}{2} \tilde{f}_{\mu}$, где $\tilde{f}_{\mu}$ из леммы $7 ; E_{k, j}=\{|z|=1\}$. Тогда с учетом свойств функции $\tilde{f}_{\mu}$ для последовательностей выполняются все утверждения леммы 8. Отсюда с учетом замечания 1 следует утверждение теоремы.

Работа выполнена при частичной финансовой поддержке РФФИ (проект № 14-07-91370).

\section{Библиографический список}

1. Смирнов В. И., Лебедев Н. А. Конструктивная теория функций комплексного переменного. М. : Наука, 1964. 440 c.

2. Привалов $A$. A. О расходимости интерполяционных процессов на множестве второй категории // Мат. заметки. 1975. Т. 18, № 2. С. 179-183.
3. Шаталина А. В. Расходимость интерполяционных процессов Лагранжа на единичной окружности / Сарат. гос. ун-т. Саратов, 1990. 30 с. Деп. в ВИНИТИ 19.07.1990, № 4060-В90.

4. Прахар К. Распределение простых чисел. М. : Мир, 1967. 513 c.

\section{Everywhere Divergence of Lagrange Processes on the Unit Circle}

\section{S. V. Tyshkevich ${ }^{1}$, A. V. Shatalina ${ }^{2}$}

Saratov State University, 83, Astrakhanskaya str., 410012, Saratov, Russia, tyszkiewicz@yandex.ru, teh-plast@bk.ru We study the convergence of Lagrange interpolation processes in the closed unit disk. Choosing a matrix with a certain distribution of interpolation nodes allowed to construct the set, completely covering the unit circle, and the function for which the process diverges everywhere on this set.

Key words: interpolation, Lagrange polynomials.

This work was supported by the Russian Foundation for Basic Research (project no. 14-07-91370). 


\title{
References
}

1. Smirnov V. I., Lebedev N. A. Functions of a Complex Variable: Constructive Theory. London, Iliffe Books Ltd., IX, 1968, $488 \mathrm{pp}$.

2. Privalov A. A. Divergence of nterpolation processes on sets of the second category. Math. Notes, 1975, vol. 18, no. 2, pp. 692-694.

3. Shatalina A. V. Divergence of Lagrange Processes on the Unit Circle. Dep. v VINITI [Dep. in VINITI], Saratov State University, no. 4060-B90, 19.07.1990, 30 p. (in Russian).

4. K. Prachar. Raspredelenie prostyh chisel [The Distribution of Prime Numbers]. Moscow, Mir, 1967. 513 p. (in Russian).

УДК 517.95; 517.984

\section{КЛАССИЧЕСКОЕ РЕШЕНИЕ МЕТОДОМ ФУРЬЕ СМЕШАННЫХ ЗАДАЧ ПРИ МИНИМАЛЬНЫХ ТРЕБОВАНИЯХ НА ИСХОДНЫЕ ДАННЫЕ}

\begin{abstract}
А. П. Хромов ${ }^{1}$, М. Ш. Бурлуцкая ${ }^{2}$
${ }^{1}$ Доктор фризико-математических наук, профрессор кафедры диффреренциальных уравнений, Саратовский государственный университет им. Н. Г. Чернышевского, KhromovAP@info.sgu.ru

${ }^{2}$ Кандидат фризико-математических наук, доцент кафредры математического анализа, Воронежский государственный университет, bmsh2001@mail.ru

В статье дается новое краткое доказательство теоремы В. А. Чернятина о классическом решении методом Фурье смешанной задачи для волнового уравнения с закрепленными концами при минимальных требованиях на начальные данные. Далее, рассматривается подобная задача для простейшего фрункционально-дифрференциального уравнения первого порядка с инволюцией в случае закрепленного конца, и также получаются результаты окончательного характера. Эти результаты получаются благодаря существенному использованию идей А. Н. Крылова по ускорению сходимости рядов, подобных рядам Фурье. Без доказательства приводятся результаты и для других схожих случаев смешанных задач.
\end{abstract}

Ключевые слова: смешанная задача, метод Фурье, инволюция, классическое решение, асимптотика собственных значений и собственных фрункций, система Дирака.

\section{ВВЕДЕНИЕ}

Настоящая публикация приурочена к 150-летию со дня рождения выдающихся отечественных ученых В. А. Стеклова (1884-1926) и А. Н. Крылова (1883-1945), внесших весомый вклад в решение смешанных задач методом Фурье.

Обоснование метода Фурье в задачах математической физики традиционно опирается на доказательство равномерной сходимости ряда, представляющего формальное решение задачи, и рядов, полученных его почленным дифференцированием нужное число раз.

Приведем мнение В. А. Стеклова, впервые давшего строгое обоснование метода Фурье: «Необходимость доказывать равномерную сходимость рассматриваемых рядов вытекает из самой сущности метода Ляме - Фурье (Эйлера - Бернулли), дающего выражение искомой функции в виде бесконечного ряда, просуммировать который или преобразовать к виду, удобному для дифференцирования, не представляется возможным» [1, с. 224].

Эта точка зрения сделала метод Фурье очень популярным, было проведено огромное количество исследований и достигнуты значительные успехи.

Информация обзорного характера содержится, в частности, в книгах И. Г. Петровского, В. И. Смирнова, О. А. Ладыженской и В. А. Ильина, В. А. Чернятина [2-7].

Приведем один такой результат из [2]. Рассматривается задача

$$
\begin{gathered}
u_{t t}=u_{x x}-q(x) u, \quad x \in[0, \pi], \quad t \in(-\infty,+\infty), \\
u(0, t)=u(\pi, t)=0, \\
u(x, 0)=\varphi(x), \quad u_{t}(x, 0)=\psi(x) .
\end{gathered}
$$

Теорема 0.1 [2, с. 190]. Если $q(x) \in C[0, \pi]$ и вещественна, $\varphi(x) \in C^{3}[0, \pi], \psi(x) \in C^{2}[0, \pi]$,

$$
\varphi(0)=\varphi(\pi)=\varphi^{\prime \prime}(0)=\varphi^{\prime \prime}(\pi)=0,
$$

